\title{
Merckel cell carcinoma of the skin treated with somatostatin analogue and mTOR inhibitor exhausted after primary surgery, adjuvant radiotherapy and palliative chemotherapy
}

\author{
Slovacek L \\ Department of Clinical Oncology and Radiation Therapy, Charles University Hospital and Faculty of Medicine, \\ Hradec Kralove, Czech Republic. Ladislav.Slovacek@seznam.cz
}

\begin{abstract}
Merkel cell carcinoma is a highly aggressive neuroendocrine skin tumor. This type of tumor is primarily based on Merkel cells located in the basal layer of the epidermis. The tumor occurs predominantly in elderly individuals (average age 69 years) and has a strong tendency to local recurrence and locoregional metastasis. Diagnosis of the tumor is based on histological and immunohistochemical examination. The therapy is radical surgery followed by adjuvant radiotherapy to the site of the primary tumor and regional lymph nodes. The authors describe a case report of Merkel cell carcinoma of the skin treated with somatostatin analogue and mTOR inhibitor exhausted after primary surgery and adjuvant radiotherapy and paliative chemotherapy (Fig. 2, Ref. 17). Text in PDF www.elis.sk.

Key words: carcinoma, Merkel cells, somatostatin analogue, mTOR inhibitor.
\end{abstract}

Merkel cell carcinoma (MCC) is a rare primary neuroendocrine skin tumor associated with a poor prognosis (1). This type of tumor is primarily based on Merkel cells located in the basal layer of the epidermis. The tumor occurs predominantly in elderly individuals (average age 69 years) and has a strong tendency to local recurrence and locoregional metastasis. Diagnosis of the tumor is based on histological and immunohistochemical examination. The therapy is radical surgery followed by adjuvant radiotherapy to the site of the primary tumor and regional lymph nodes (1).

\section{Case report}

70-year-old female patient with tumor triplicity (papillary tonsilar carcinoma, lung carcinoma and Merkel cell carcinoma) was treated from January 2008 in Cancer Center (Fig. 1). The patient underwent radical surgery of the right elbow, followed by adjuvant radiotherapy up to a total dose of $54 \mathrm{~Gy}$ in 27 fractions. After three months there was a progression of the tumor within the meaning of the new bearings on the right arm (Fig. 2). Palliative chemotherapy based on cisplatin and etoposide was indicated. A total of five chemotherapy cycles were given, after which a regression of the disease was achieved. Another local disease progression on the right arm occurred approximately after nine months, subsequently

Department of Clinical Oncology and Radiation Therapy, Charles University Hospital and Faculty of Medicine, Hradec Králové, Czech Republic

Address for correspondence: L. Slovacek, MD, PhD, Department of Clinical Oncology and Radiation Therapy, Charles University Hospital and Faculty of Medicine, Sokolska 581, CZ-500 05 Hradec Kralove 5, Czech Republic.

Phone: +420.495.832176, Fax: +420.495.832180

Acknowledgements: Supported by MH CZ - DRO (UHHK, 00179906). re-irradiation of right arm was applied and then additional five cycles of palliative chemotherapy based on cisplatin and etoposide in reduced doses of both by $25 \%$ due to haematological toxicity (pancytopenia) were administered leading to stable disease. After about three months there is a significant local tumor progression not only on he right arm, but also on the right side of the neck and chest wall. Due to exhaustion of the possibilities of cancer therapy the patient was considered for biological treatment. Based on the positive octreoscan somatostatin analogue therapy was idnicated (lanreotide $120 \mathrm{mg}$ intramuscularly at intervals of two weeks). After three applications of lanreotide clinically significant progression in number of active sites on the right upper extremity and right chest wall were observed. It was therefore changed to a combination of lanreotide with mTOR inhibitor (everolimus at a dose of $10 \mathrm{mg}$ daily). After one month of combined biological therapy, tumor progression was observed again. Cancer treatment was finally stopped. The patient after giving consent was included in a programme of palliative cancer care to preserve as the best possible level of quality of life.

\section{Discussion}

Merkel cell carcinoma (MCC) is a rare primary neuroendocrine skin tumor associated with a poor prognosis (1). It is thought to be derived from Merkel cells of the skin, but it has also been proposed that the cell of origin may be a primitive pluripotent epidermal cell $(2,3)$. MCC typically presents as a firm, pinkishred nodular or plaque-like exophytic mass. The most common locations are in sun-exposed skin of the head, neck or extremities (4). Wide local excision is the mainstay of treatment (5), although radiotherapy has often been used as adjuvant or definitive treatment (6-10). As 


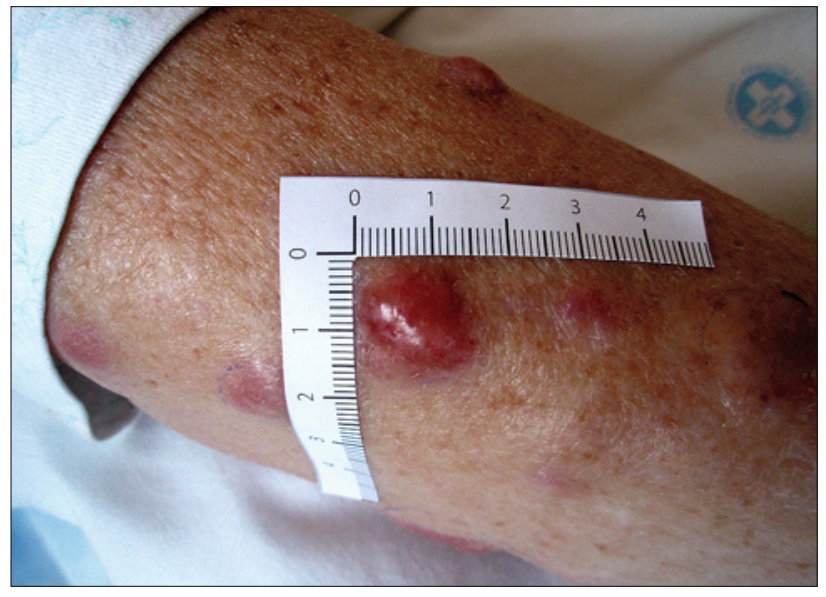

Fig. 1. Merkel cell carcinoma diagnosed in January 2008.

with melanoma, depth of invasion is a prognostic feature (11-13). MCC is locally aggressive, local recurrence rates are between 25 and $100 \%$ following surgical excision and the mortality rate of MCC is between 30 and $60 \%$ (4). Traditional management has been surgical resection as the mainstay of local therapy. However, standardized adjuvant therapy is less well defined. The standardized treatment consisted of Carboplatinum (day 1) and Etoposide (days 1-3) with concurrent radiotherapy (5000 cGy at 200 cGy/ $\mathrm{fx}$ with wide margins) followed by two additional cycles of chemotherapy (14). Viola et al (14) reported that surgical excision with or without lymph node dissection, followed by postoperative radiotherapy in stage II disease, is the standard treatment of non-metastatic disease. The role of adjuvant chemotherapy is still controversial. In patients with metastatic disease, chemotherapy regimens active in small cell lung cancer are generally used. The combination of cyclophosphamide, doxorubicin and vincristine (CAV) has an overall response rate of $75 \%$, whereas the response rate of etoposide in combination with cisplatin or carboplatin is $60 \%$. Experience with other therapeutic agents, such as tumor necrosis factor, interferon and octreotide is scanty (14). Meier et al (15) report the case of an 83-year-old woman with recurrent MCC of the left cheek. The primary tumor and several relapses were treated with surgery and locoregional radiotherapy. After the 3rd relapse, she was treated 4 times with 90Y-DOTATOC and two complete remissions were achieved. The fourth administration after the 2nd relapse was ineffective and conventional chemotherapy was started. There were no side effects of the 90Y-DOTATOC. 90Y-DOTATOC is a novel radiolabeled somatostatin analogue containing the active octapeptide of somatostatin. It is very well tolerated and offers the option of treating somatostatin receptorpositive tumors by targeted radiotherapy (15). Also, Fakiha et al (16) recently treated 87-years-old female patient presenting with a disseminated non-operable MCC by lanreotide (somatostatin analog), with a complete remission of the disease and a follow up of 17 months. Novel therapies use of imiquimod (an immune modulator that activates TLR7 cells on the skin surface) cream plus radiotherapy (5040 cGy in 28 fractions) has been used anecdotally

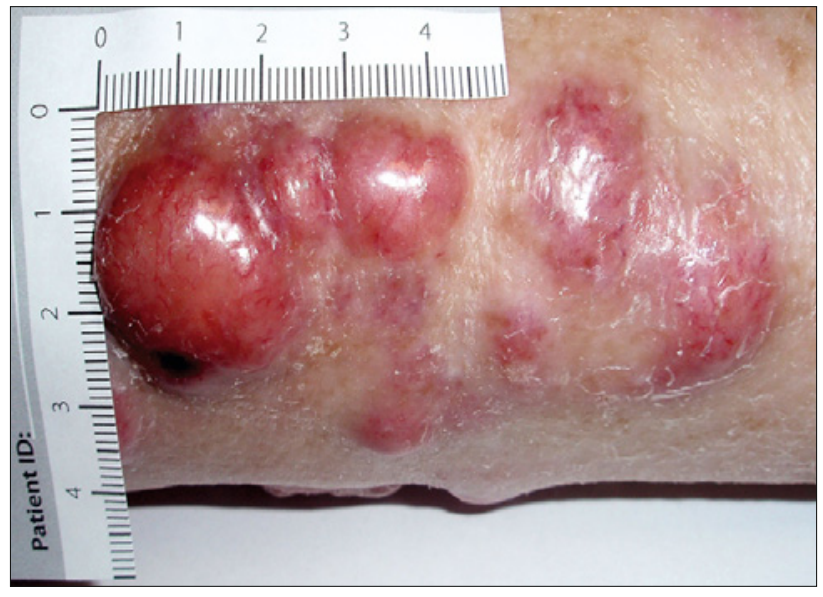

Fig. 2. The progression of the tumor within the meaning of the new bearings on the right arm after three months.

as has hyperthermia with radiotherapy with limited success. Both groups demonstrated local control, however adjuvant radiotherapy was delivered in both cases and the margins of resection were histologically negative (17).

\section{Conclusion}

In conclusion, we describe a case report of Merkel cell carcinoma of the skin treated with somatostatin analogue and mTOR inhibitor exhausted after primary surgery and adjuvant radiotherapy and paliative chemotherapy. The case report is interesting because of 3 reasons: 1) attention is focused onone type of neuroendocrine tumor of skin with an incidence in elderly patients, including its aggressive behavior in the sense of local recurrence or locoregional metastases. 2) it describes the current possibilities of treatment of this cancer type, including the possibility of biological treatment. 3) emphasis is on intensive nursing care as an essential component of a comprehensive therapeutic intervention for patients with this type of disease.

\section{References}

1. Miranda S, Gbaguidi X, Carvalho P, Chassagne J. Merckel cell carcinoma: the impact of multidisciplinary management. J Nutr Health Aging 2013; 17(2): 196-198.

2. Thakuria M, LeBoeuf NR, Rabinowits G. Update on the biology and clinical management of merkel cell carcinoma. Am Soc Clin Oncol Educ Book 2014; 34: e405-410.

3. Feng H, Shuda M, Chang $\mathbf{Y}$ et al. Clonal integration of a polyomavirus in human Merkel cell carcinoma. Science 2008; 319: 1096-1100.

4. Hausschild A, Garbe C. Cutaneous neuroendocrine carcinoma (Merkel cell carcinoma). Quality Assurance Committee of the German Society of Dermatology and Professional Organization of German Dermatologists. Hautarzt 1998; 48: 27-29.

5. Eng TY, Boersma MG, Fuller CD et al. Treatment of Merkel cell carcinoma. Am J Clin Oncol 2004; 27: 510-515. 
6. Mortier L, Mirabel X, Fournier C et al. Radiotherapy alone for primary Merkel cell carcinoma. Arch Dermatol 2003; 139: 1587-1590.

7. Veness M, Foote M, Gebski V et al. The role of radiotherapy alone in patients with Merkel cell carcinoma: reporting the Australian experience of 43 patients. Int J Radiat Oncol Biol Phys 2010; 78: 703-709.

8. Gillenwater AM, Hessel AC, Morrison WH et al. Merkel cell carcinoma of the head and neck: effect of surgical excision and radiation on recurrence and survival. Arch Otolaryngol Head Neck Surg 2001; 127 : 149-154.

9. Lewis KG, Weinstock MA, Weaver AL et al. Adjuvant local irradiation for Merkel cell carcinoma. Arch Dermatol 2006; 142: 693-700.

10. Mojica P, Smith D, Ellenhorn J. Adjuvant radiation therapy is associated with improved survival in Merkel cell carcinoma of the skin. J Clin Oncol 2007; 25: 1043-1047.

11. Mott RT, Smoller BR, Morgan MB. Merkel cell carcinoma: a clinicopathologic study with prognostic implications. J Cutan Pathol 2004; 31: 217-223.

12. Gupta SG, Want LC, Penas PF et al. Sentinel lymph node biopsy for evaluation and treatment of patients with Merkel cell carcinoma. Arch Dermatol 2006; 142: 685-690.
13. Allen PJ, Bowne WB, Jaques DP et al. Merkel cell carcinoma: Prognosis and treatment of patients from a single institution. J Clin Oncol 2005; 23: 2300-2309.

14. Viola G, Visca P, Bucher S, Migliano E, Lopez M. Merkel cell carcinoma. Clin Ter 2006; 157: 553-559.

15. Meiera G, Waldherrb C, Herrmanna R, Maeckeb H, MuellerBrandb J, Plessa M. Successful Targeted Radiotherapy with 90Y-DOTATOC in a Patient with Merkel Cell Carcinoma. Oncology 2004; 66: $160-163$.

16. Fakiha M, Letertre P, Vuillez JP, Lebeau J. Remission of Merkel cell tumor after somatostatin analog treatment. J Cancer Res Ther 2010; 6: 382-384.

17. Trombetta M, Packard M, Velosa C, Silverman J, Werts D, Parda D. Merkel cell tumor of the skin treated with localized radiotherapy: are widely negative margine required? Rare Tumours 2011; 3 (1): $36-38$.

Received April 13, 2011. Accepted June 9, 2014. 